\title{
CERTAIN ISSUES AND CHALLENGES OF SCIENCE AND RESEARCH IN THE COVID-19 PANDEMIC
}

\author{
RADOSAVLJEVIC Zivota ${ }^{1}$, ANDJELKOVIC Aleksandar ${ }^{2}$, KASTRATOVIC Edita ${ }^{3}$ \\ 1 Faculty of Business Studies and Law,Belgrade (SERBIA) \\ 2 Faculty of Business Studies and Law, Belgrade (SERBIA) \\ 3 Faculty of Business Economics and Entrepreneurship, Belgrade (SERBIA) \\ E-mails: zivota.radosavljevic@fpsp.edu.rs; aca.andjelkovic@fpsp.edu.rs; edita.kastratovic@vspep.edu.rs
}

\begin{abstract}
The crisis of the COVID-19 pandemic is probably one of the biggest crises that has affected civilization in the last hundred years. This refers to the number of infected and dead, but also to the economic, psychological, political, moral and other spheres.

Science and scientific research during the pandemic was mainly focused on finding ways to prevent and treat the C-19 virus. Within medical science, epidemiology was treated separately during the pandemic, as a branch of medicine, while other sciences were neglected or put in the background, which limited the potentials of medical science in the $\mathrm{C}-19$ pandemic.

Analyzes show that a small number of scientific papers and research have focused on non-medical sciences and the place and role of non-medical professions in the fight against C-19, which is illogical, given that a pandemic is not just a medical phenomenon but a multidisciplinary problem that should be answered, with synchronized use of information, economic, organizational management, ethical and other sciences.

It is shown that the impact of the pandemic on science and scientific research has not been investigated globally, nor at the level of Serbia, ie whether and to what extent the pandemic has affected the dynamics, aggravating or facilitating conditions for science and scientific research. This paper also discusses the problems of science and scientific cooperation during the Covid-19 pandemic and the impact of the COVID-19 pandemic on science, scientific research and cooperation, and cooperation between scientific researchers at the international level.

The aim of this paper is to point out some difficulties and obstacles in scientific work and cooperation and to suggest possible solutions.
\end{abstract}

Keywords: Pandemic, Problems of Science in a Pandemic, International Scientific Cooperation and $\mathrm{C}-19$

JEL: $I 15$

DOI: 10.5937/intrev2103122R

UDC: 616.98:578.834]:001.891

COBISS.SR-ID 55111177 


\section{INTRODUCTION}

The management of the pandemic crisis has been inconsistent since its inception; in many elements it was unclear and contrary to scientific and logical conclusions and actions. Although crisis management belongs to managerial organizational sciences, this work has been wrongly handled by medical professionals who have not been trained in crisis management. Thus, the world on a global level came to a situation where the pandemic is managed by medical experts through the institute of crisis headquarters, who during medical education had almost no contact with sciences such as organization, management, crisis management, operational research, system theory, chaos theory, etc. These headquarters in transition and underdeveloped countries represented experts who were supposed to "cover" the decisions of politicians, who often dominated the crisis headquarters and who thought more about the rating of their political options than about the health of the population. It is evident from the above that science in the pandemic came under the great influence of politics and that even the medical profession was subordinated to politics, ie to politicking.

The second fact that needs to be considered and investigated is whether and to what extent public health at the global level was prepared for the pandemic. This is all the more so because pandemics are occasional and temporary, and states usually have optimistic scenarios in which the crises will not appear in the near future. Attitudes should also be reconsidered which claim that expenditures for the preparation of something uncertain in the future are irrational and that these funds should be redirected to other activities.

There is no need to explain just how wrong this is, because it turns out that the damage from the C-19 pandemic was far greater than investing in infrastructure, technology, but also in human resources that would serve to protect the population from infection and for the treatment of infected. Even when these capacities are not used, they are the most cost-effective, such as firefighters and medical experts, who are most useful when they do not have work to perform.

The high degree of politicization of the pandemic at the global level has led to the primary place given to projects and investments that have increased the rating of the political elite in power, putting the present, not the future, in the foreground. The basic hypothesis in this paper is that the COVID-19 pandemic creates scientific tensions and negatively affects science and scientific research, and that the pandemic objectively reduces scientific potential.

However, the real fact is that in crisis situations and when a person is in danger of life and health, people unite, they are more willing to cooperate and help, which has been confirmed throughout history, because a person as an individual does not have much strength or knowledge to counteract natural and other dangers, which are becoming more complex and challenging. What a person has is the ability to socialize and act together, to cooperate with other people, in order to overcome individual weaknesses and respond to the challenges of natural disasters, including the pandemic crisis.

It is to be expected that those sides of sciences and scientific research that have proven to be poor will be eliminated, both in future pandemics and in normal times, and that cooperation in the scientific field will be strengthened, especially when it comes to medical science, which deals with health and people's lives.

\section{SCIENTIFIC COOPERATION AT THE GLOBAL LEVEL}

It is known that everything that exists on the planet earth is the result of science, ie of new knowledge and insights, which man acquired by observing nature and the world around him and its functioning, from which he drew certain laws. The crisis of the COVID-19 pandemic is a great challenge for individuals, as well as countries, in order to adequately respond to it. "Successful international scientific cooperation encourages discovery, innovation and economic development. Successful collaborations, such as CERN, the Human Genome Project, and LIGO, use international talent to overcome the limitations of individuals. In other words, a successful response to the COVID19 pandemic is possible through the so-called mega scientific endeavors and projects" [1].

International scientific cooperation is a condition for a successful fight against pandemics. The readiness of scientific power houses such as the United States and China to cooperate in the fight against C-19 has put aside decades of conflicts. Both countries, but also other developed countries, have 
engaged in mega research, taking into account national security, or other national goals and interests. Analyzes have shown that the United States has three reasons to participate in large-scale international scientific research. [2]

- Participation enables inspiring discoveries, which are otherwise impossible,

- Improve life, and

- Promoting international understanding.

It is clear that throughout history, people have tried to discover inspiring secrets, which are on the level of fantasy, because such discoveries occupy the imagination around the world. This is impossible without international scientific cooperation and financial support for such mega scientific projects.

Improving the quality of life is the goal of any scientific research in this context. In addition to man trying to solve many world mysteries, international scientific cooperation and research make a significant social contribution as a whole, such as the discovery of new technologies for diagnostics and therapy in medical science.

International mega-scientific cooperation contributes to international understanding, because scientists are people who are ready to overcome national borders and other barriers, ie they are ready to understand the world and what is happening in it, and especially to overcome differences regarding national, ethical and other characteristics.

Medical and pharmaceutical science at the global level reacted quickly in the pandemic by creating vaccines that became medical brands, of which more or less the majority of the population globally knows and uses them. Without science and collaboration with researchers at universities from different countries and cultures, people's lives and health would be at stake in a relatively short time. Cooperation between academia and the pharmaceutical industry has been shown to be good in many parts of the world, leading to 16 vaccines being approved globally, with another 9 in full clinical trials [3].

The crisis of the COVID-19 pandemic showed a high degree of cooperation between scientists of two mutually opposing scientific powers in the world: the United States and China. Their cooperation in the initial phase of the pandemic was intensive, and the United States later decided to cooperate more with scientists from Great Britain. The phenomenon of increased interest of scientists and the formation of scientific teams for the research of the C-19 virus also appeared in the pandemic, which was a surprise. It turned out that "within a few months of collaboration, a large number of research papers were published showing protein interactions and other characteristics of CAES-COV-2" that helped identify candidates for the drug being tested for the virus suppresion." It turned out that there was a high degree of cooperation between scientists in this work, which should be studied and the experiences from the above should be transferred to the research of other diseases, as well as to other activities [4].

It is noticeable that scientists and researchers felt more pressure during the pandemic to share and exchange knowledge and information, as well as the results of scientific research. It turned out that the legislation regarding the protection of intellectual rights and property in the pandemic also showed its weaknesses in terms of keeping secrets of its results, in order to achieve a greater financial effect. The closure, or insufficient exchange of knowledge about the virus between scientists and researchers at the international level, has also led to less visibility of science centers and their experts, which has weakened international cooperation and science as well.

Related to the previous is the commitment of the scientific community at the global level to make huge individual, or institutional depots of knowledge that are stored, available to the scientific community and the general public, through the so-called "Open Science". The C-19 pandemic encouraged the development of the idea of open science, which would be "concretely implemented through a number of basic open science practices, such as: Open Access, Open Source, Open Data and Open Peer-Rewiew, meaning that all scientific communication is free available with full reuse rights. An open approach would also include papers that have not been reviewed and that have not been published in scientific journals, but which contribute to the transparency of science development, although they may have certain errors and shortcomings. However, it should be borne in mind that there is no absolute inaccuracy in unpublished and unreviewed papers, but that scientific claims and laws in them, as in peer-reviewed papers, should be constantly checked and solutions sought that will have a higher degree of probability, or lower production costs, etc. The idea of open science stimulates scientific debate, which would benefit the subjects who manage the pandemic crisis in the fight against COVID-19 and the exchange of different ideas [5]. 
Experience from the C-19 pandemic shows that at the time of the COVID-19 pandemic, the distribution of research was not even. Richer countries and science centers with larger research budgets were mainly engaged in scientific research. It turned out that during the pandemic, "most large international clinical trials were conducted by more developed countries, including the treatment of vaccines, which could be given in a hospital setting." This has prolonged, but also increased the costs of finding vaccines, tests and other antiviral drugs. The price listed in the number of infected or dead was paid by countries with low or medium income and development. If there had been greater international scientific cooperation, as well as humanity, many problems in the fight against the C-19 virus would have been solved faster, and in a more effective way.

From the above, it can be concluded that cooperation in science and in mega scientific research work between scientific institutions of different countries is necessary in order to respond to the increasingly complex challenges facing the world. Experience shows that the world would be deprived of many solutions that have been provided if there were no cooperation between well-known universities in the world, but also it there was no financial support from countries, or international financial and other institutions.

\section{COOPERATION OF SCIENTISTS AND RESEARCHERS DURING THE COVID-19 PANDEMIC}

Related to the previous issue is the cooperation of scientists and researchers during COVID-19 on a global level. Closedowns, restrictions and bans have had a negative impact on collaboration between scientists and researchers, requiring the search for alternative ways to collaborate. The way out was found in the application of information and communication technologies that enabled research to be performed remotely. Yet many perennial links in international research have either slowed or broken, with little prospect of renewing them, either due to their disappearance, or the fluctuation of scientists and researchers and their going to other scientific institutions at home and abroad.

Research conducted by Nature shows that the cooperation between scientists, researchers and universities at the time of the pandemic was dominated by the topic of the distribution of intellectual property, ie ownership of data and information. This was surprising to many, as many believed that universities would not think so much about the monetization of science. Many in "some industries have created the perception that universities produce science and leave it to the industry to commercialize it." What is new in relation to the previous decades in which the pandemic appeared is that the universities tried to maximize the return of funds from science, creating competition between universities and companies ".

Another problem in the COVID-19 pandemic crisis concerns access to data, because researchers did not have access to data in emergencies, especially data from the pharmaceutical industry that are not commercially vulnerable. This has significantly reduced the speed of researchers. The issue of intellectual property is otherwise problematic even in normal conditions, because in the world of informatics and developed information and communication technologies, it is difficult to determine who the author of an idea or product is and who owns the property that should be commercialized. Experience shows that a significant contribution to the development of vaccines and other scientific products was made even by those who failed in certain experiments, or their result was of lower degree of reliability in the treatment of infection, because they showed which direction not to take, which is also contribution to science.

The criteria by which the quality of scientific papers was evaluated during the pandemic is also problematic, because the value of scientific papers was dominated by the Index of Scientific Citation, and the number and scope of scientific papers in the databases of international journals. It turned out that the National Policy of China, ie their Government in 2020 stated that "Chinese researchers should be evaluated less on the basis of the scope of their work in databases of international journals, and more on the basis of the quality of papers and that the results achieved and published in China should have been taken into account". [6]

It turns out that scientific cooperation during the COVID-19 pandemic was different. For some, the pandemic made research and collaboration difficult, while others in the crisis sought a way to overcome the obstacles that research made difficult. Here, as in other fields, it has been shown that "Those who 
want cooperation in the field of science, especially in times of crisis, will find ways to achieve that, while those who do not want will find an excuse or reason to explain why it is impossible."

Scientific cooperation had different dynamics during the pandemic. At the beginning of the pandemic, researchers were more focused on researching health infections and finding a vaccine, ie more focused on the medical sciences, while other areas, even in national budgets, gained less importance. It turns out that "Never before, say scientists, have so many researchers focused on one topic at the same time and with great urgency to the extent that almost all other research has stopped or has been interrupted."[7]

According to the analysis of bibliometric data, it can be stated "that international cooperation was less common in the papers related to COVID-19 in 2020 than it was in the research of other coronaviruses in previous years." It is evident that with the spread of the pandemic, the number of papers whose authors were from the same country grew and that these papers occupied most of the literature on COVID-19. Looking at the international cooperation as a whole in science related to COVID-19, it can be concluded that it was at the level of previous years for similar research. It is clear that such papers have less potential, especially since individual authors even appear from the same scientific institution.

The data show that in science and research there is a trend of weakening international cooperation and that it is often influenced by geostrategic relations between countries, which has made science increasingly part of politics. Research shows that cooperation between scientific research centers and institutions between the US and China, as the two countries with the strongest scientific potential, has stagnated since 2017. When we take into consideration the well-known metaphor: "that science stands on the shoulders of giants", it can be assumed with high probability that the indicated trend will have a negative impact on the development and advancement of science and research.

With the strengthening of geopolitical tensions, and especially with the issue of straining relations on the issue of Taiwan and Hong Kong, these trends will continue, which will reduce the achievements of science and research. It turns out that the geopolitical relations between the great powers will directly affect the cooperation of science and the results of research, but also the time of arrival of new scientific information. [8]

A special challenge during the pandemic in scientific research was posed by women scientists, researchers and collaborators. Closing or reduced use of the potential of the institutions in which they worked, but also other institutions such as schools, business organizations that switched to work from home, made it difficult to engage in science and research. The closure of kitchen and restaurant facilities has forced women scientists to do housework related to the normal functioning of the household and family, such as helping children master distance learning programs, preparing food for family members, commitment to older family members and all that in small and small families. not conditional spaces, away from laboratories and other advanced technologies that exist in scientific research centers. In this regard, Vice President for Academic and Governmental Relations at the scientific journal Elsevier, Leslie Thompson states: "At the individual level, women face special challenges as members of cooperation and that the representation of women in scientific publications in 2020 has shown that women have less number of papers, compared to men, and at the same time a smaller network of international collaborators." [9].

In the crisis of the COVID-19 pandemic, the difference between male and female researchers has noticeably increased. This difference has existed before, but COVID-19 made this situation even worse. Women, scientists and researchers in the pandemic have lost their primary authorial role and appear deeper on the list of authors. The situation is the same when it comes to databases where studies and research results are registered. One of the reasons for this situation, which was discussed, is that women have taken on a large part of the responsibilities of families at the expense of scientific research work, especially in countries and households that are not technically equipped to replace women's work [9].

\section{CONCLUSION}

This paper has pointed out the importance of science and research for solving increasingly complex and challenging problems, ie some issues that reduce its efficiency. It is noticeable that on a global level, a greater degree of cooperation is needed between scientific research institutions from different 
countries and different cultures. The key scientific superpowers in the world, after many years of geostrategic conflicts, have shown a certain degree of scientific cooperation in the crisis of the COVID19 pandemic. In mega science and scientific research, the main role is played by the United States, but in research funded by the United States, dozens of countries from different continents participate. Thus, the pandemic, in addition to its negative sides, has an increased need for international scientific cooperation and funding, although maneuvering opportunities have made this cooperation difficult due to numerous closures and bans.

The pandemic had a negative impact on personal researchers, due to the closure of scientific research institutions, and the distance of researchers from scientific centers, experimental laboratories, innovation centers, etc. This problem has been partially solved, by using information and communication technologies and working remotely. The application of information and communication technologies in the global world environment is constantly increasing[10].

The work of women, who were forced to work from home due to the limitations of the work of scientific institutions, where they spent a significant part of their time serving families, was especially difficult. This negativity has been particularly evident in households that are not technically equipped enough and where women have to expose themselves to physical exertion and spend time on household, rather than scientific research projects.

The paper also points out the need for a different evaluation of scientific research papers and a different approach to the protection of intellectual property, and the need for all knowledge to be considered a global good available to everyone, and for authors who have combined and realized ideas to be funded differently. This is necessary primarily in the conditions of a mass crisis, where there are large losses in people and damage to their health, but also in the so-called post pandemic pandemics. In this way, the pharmaceutical industry and scientists would become less commercialized and this would also increase trust in the products they finalize through the medical profession.

\section{REFERENCES}

[1] "Exploring the Future of International Larege-Scale Science", American Academy of Art and Science, june 2021.

[2] Imperatives for International Large-Scale Science, American Academy of Art and Science, 2021., https://www.amacad.org/publication/international-large-scale-science pristup 10.08.2021.

[3] Kowalski C. (2021) COVID has shown the power of science-industry collaboration", Nature

[4] Fabius, J.M., Krogan Nj. (2021)“Cell” 184, 2271-2275,

[5] Munafo MR et al.(2017) “A manifest for reproducible science”, Nat Hum Behav, pp. 1-9.

[6] OECD (2021)How will Covid-19 reshape science, tehnology and innovation, OECD.

[7] Apuzzo M., Kirkapatrick D.(2020) "Covid-19 Changed How the World Does Science, Together", www-nytimes-com.translate.goog/2020/04/01/world/europe/coronavirus-science-research. coperation.html?), pristupljeno 30.07.2021.).

[8] Research collaborations bring big rewards: the world needs more", Natural 594, 2021, pp.301-302.

[9] Gender in the Global Research Landscape, Elsevier, 2020.https://www.elsevier.com/_data/assets/pdf_file/0008/265661/ElsevierGenderReport_final_ for-web.pdf pristup 05.08.2021.

[10]Kalicanin K., Brdar I.,Vesic T. (2020) „Education is becoming digital-the youth attitudes towards online teaching durinh the COVID-19 pandemic, International Review, No. 1-2,Faculty of Business economics and Entrepreneurship

\section{Article history:}

Received 15 November 2021

Accepted 21 December 2021 